\title{
Diacronie
}

Studi di Storia Contemporanea

$\mathrm{N}^{\circ} 7,3$ | 2011

«Spagna Anno Zero»: la guerra come soluzione

\section{Guerra civile e architettura spagnola moderna: la fine di un sogno}

\section{Gemma Domènech Casadevall}

\section{Q OpenEdition \\ 1 Journals}

Edizione digitale

URL: http://journals.openedition.org/diacronie/3384

DOI: $10.4000 /$ diacronie.3384

ISSN: 2038-0925

Editore

Association culturelle Diacronie

Notizia bibliografica digitale

Gemma Domènech Casadevall, « Guerra civile e architettura spagnola moderna: la fine di un sogno », Diacronie [Online], $N^{\circ} 7,3$ | 2011, documento 18, online dal 29 juillet 2011, consultato il 10 décembre 2020. URL : http://journals.openedition.org/diacronie/3384 ; DOI : https://doi.org/10.4000/diacronie. 3384 


\title{
Diacronie
}

\section{8/}

\section{Guerra civile ed architettura spagnola moderna: la fine di un sogno}

\author{
Gemma DOMÈNECH CASADEVALL *
}

Nei primi anni Trenta in Catalogna si afferma una chiara comunione di interessi all'interno di un gruppo di giovani architetti mossi da inquietudini verso la modernizzazione e percorsi dall'ideologia politica repubblicana e progressista. Insieme cercano di modificare le condizioni di vita dei cittadini. Politici e architetti condividono la preoccupazione per il modo di abitare delle classi lavoratrici, per la pianificazione delle città, il design di uso pubblico sociale e di tutela dei beni culturali. Dopo la guerra, come conseguenza di questa identificazione tra il movimento d'architettura innovativa e la Repubblica, l'architettura moderna viene respinta e molti di suoi attori subiranno rappresaglie nelle forme dell'reclusione, interdizione professionale, o dell'esilio.

\section{Introduzione}

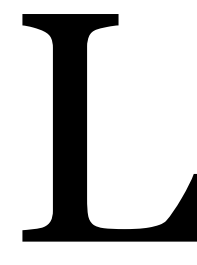

a notte del 26 gennaio 1939 Emili Blanch attraversava la frontiera francese a piedi, iniziando un esilio di nove anni. L'uomo che era stato l'architetto responsabile delle costruzioni civili della Generalitat di Catalogna nella regione di Girona durante la Repubblica e rappresentante emergente della architettura razionalista, abbandonava la Catalogna senza attendere la risoluzione delle istituzioni della giustizia franchista.

In quei giorni, il fascismo vincitore iniziava un'implacabile attività repressiva e punitiva con la chiara intenzione di soffocare il dissenso. Oltre alle migliaia di morti e d'incarcerati, chiunque era legato in qualsiasi modo con la politica democratica fu sottoposto ad un processo dal Tribunal de Responsabilidades Políticas. 
Parallelamente, la fazione vincente aveva avviato un intenso lavoro di epurazione volto a giustiziare i professionisti che avevano lavorato per la Repubblica. Saranno processi civili destinati a punire e soprattutto intimidire i vinti. I funzionari, insegnanti, medici, avvocati, architetti, ecc. saranno costretti ad abbandonare la loro professione. ${ }^{1}$

Nel caso di Emili Blanch, la sua appartenenza politica al partito Esquerra Republicana e il suo lavoro per il governo della Repubblica determinano la condanna da parte del Tribunal de Responsabilidades Políticas, che impone la confisca totale dei beni, il sollevamento da ogni incarico professionale e l'esilio nei territori africani per un periodo di quindici anni. ${ }^{2}$ Inoltre, la sua attività professionale davanti alla Secció de Construccions Civils del Governo della Generalità a Girona e alla Comissió del Patrimoni Artístic sarà sentenziata dalla Comisión de Depuración constituita nel Collegio di Architetti. Nel luglio del 1942 questo Tribunale risolve la sospensione totale nell'esercizio pubblico e privato della professione in tutto il territorio nazionale, le sue possessioni e Protettorato. ${ }^{3}$

La pesante lastra di Franco copre con la sua ombra schiacciante non solo la diversità politica o la rivalità ideologica. Oblia, simultaneamente, carriere professionali che erano nel loro periodo di massimo splendore. In altri casi, ne tronca altre che nascevano. Alla fine della Guerra Civile furono molti i cittadini espulsi dal loro universo familiare e professionale, sulla via dell'esilio e sottoposti a varie forme di repressione, che includono la prigione (o la morte), l'epurazione professionale, l' inabilitazione, l'esilio o un insieme drammatico di queste varie forme di rivincita ufficiali. Emili Blanch Roig (1897-1996) è stata una delle vittime inquadrabili perfettamente in questo modello repressivo. Il suo caso, uno dei tanti, è paradigmatico di un individuo professionalmente brillante e vittima della repressione totale e sistematica del regime vittorioso, che è stato capace di rendere anonima la sua figura nella storia dell'architettura. 4

\footnotetext{
${ }^{1}$ GONZÁLEZ AGAPITO, Josep, MARQUÈS SUREDA, Salomó. La repressió del professorat a Catalunya sota el franquisme. Barcelona, Institut d'Estudis Catalans, 1996. CUETO RUIZFUNES, Juan Ignacio del, "Depuración político-social de arquitectos en la España de postguerra ». Bitácora-Arquitectura, n.13/2005, pp. 24-27.

2 Arxiu Històric de Girona. Fons Audiència Provincial de Girona, Responsabilitats Polítiques. Peces d’inventari, c. 868, exp. 852, «Juzgado Civil Especial del Tribunal Regional de Responsabilidades Políticas de Barcelona. Pieza de Embargue $N^{o}$ 252/54 de 1940 contra Emilio Blanch Roig vecino de Gerona».

3 « Orden por la que se imponen sanciones a los arquitectos que se mencionan », Boletín de la Dirección General de Arquitectura, n. 29-30/1942, pp. 18-20.

${ }^{4}$ La ricerca che circonda la figura di Emili Blanch è stata possibile grazie agli aiuti concessi nel 2010 dalla Direzione Generale de la Memòria Democràtica. Departament d’Interior, Relacions Institucionals i Participació de la Generalitat de Catalunya.
} 


\section{Una nuova architettura per un nuovo paese 5}

Nella Catalogna dei primi anni trenta c'era stata una comunione di interessi all'interno di un gruppo di giovani architetti mossi da inquietudini verso la modernizzazione e percorsi dall'ideologia politica repubblicana e progressista. La preoccupazione sociale, unita alla conoscenza della realtà europea e all'intenzione di spostare questo progresso nelle città catalane, definisce il pensiero di architetti e politici. Insieme cercheranno di modificare le condizioni di vita dei cittadini.

Emili Blanch Roig, laureato alla Scuola di Architettura di Barcelona nel 1925, fa parte di questa generazione di professionisti sensibili alle problematiche sociali del loro tempo e desiderosi di cambiare le condizioni di vita dei cittadini. Per loro, la proclamazione della Repubblica, in Catalogna e in Spagna, il 14 di aprile di 1931, è la concretizzazione di un'utopia di miglioramento e stabilizzazione per le classi lavoratrici, di urbanesimo moderno nella progettazione della città, di edilizia pubblica ad uso sociale e di protezione del patrimonio. Emili Blanch, membro dell'Esquerra Republicana de Catalunya, ha vissuto con entusiasmo il momento e così lo ha descritto nelle sue memorie: « la Repubblica è arrivata, in mezzo alla gioia incontenibile e il delirio della gente $» .^{6}$

Alcuni di questi architetti catalani, in sintonia con i colleghi di altre parti della Spagna, nell'ottobre del 1930 avevano fondato a Saragozza il Gruppo di Architetti e Tecnici Spagnoli per il Progresso dell'Architettura Contemporanea [GATEPAC]. Il gruppo catalano, però, ben presto si separa e il 13 di aprile di 1931, il giorno prima della proclamazione della Repubblica, si costituisce in GATCPAC (Gruppo di Architetti e Tecnici Catalani per il Progresso dell'Architettura Contemporanea). Il nucleo originario del nuovo gruppo è integrato con le presenze di Josep Lluís Sert, Manuel Subiño, Cristóbal Alzamora, German Rodriguez Arias, Ricardo Churruca, Josep Torres Clavé, Pere Armengou e Sixte Illescas. Ben presto il gruppo arriverà a contare più di ottanta membri7, ma saranno molti di più quelli che, senza essere associati, spesso per non vivere a Barcellona - città dove si sviluppa il gruppo, condividono i suoi ideali:

\footnotetext{
5 Questo capitolo è una sintesi del lavoro realizzato con Rosa Maria Gil grazie alla Borsa di Studi President Irla 2009 che ci è stata concessa. Cfr. DOMÈNECH, Gemma, GIL, Rosa Maria, Un nou model d'arquitectura al servei d'una idea de país, Barcelona, Fundació Josep Irla - Duxelm, 2010.

${ }^{6}$ AMGi, Fons Emili Blanch. Documentazione personale. « Guió per unes memòries », p. 16.

7 Antonio Pizza e Celia Marín pubblicano l'elenco completo. Cfr. PIZZA, Antonio, MARÍN, Celia «Els socis del GATCPAC (1931-1938) ». In PIZZA, Antonio, ROVIRA, Josep M., G.A.T.C.P.A.C. Una nova arquitectura per una nova ciutat, Barcelona, Museu d'Història de la Ciutat-Col-legi d'Arquitectes de Catalunya, 2006, pp. 42-44.
} 
modernizzare il paese alla luce delle nuove tendenze architettoniche europee. Ossia, fornire il paese di scuole, macelli, mercati, ospedali e alloggi salubri e degni.

Nel numero 25 della rivista AC. Documentos de Arquitectura, organo di diffusione del GATCPAC, gli architetti rinnovatori definiscono il loro concetto di alloggio dignitoso:

LA NUOVA ABITAZIONE deve essere circondata da tutti quegli elementi che sono necessari all'individuo, parchi, campi sportivi, giardini, club di lavoratori, scuole, biblioteche, ecc. Il divertimento quotidiano, fondamentale per il lavoratore, deve essere proporzionato rispetto a questi elementi, situati intorno agli edifici destinati a rispettare rigorosamente questa funzionalità: abitare. ${ }^{8}$

Nel mese di giugno del 1932, il governo della Generalità commissiona il primo incarico al GATCPAC: la costruzione di 10 case popolari nel quartiere di Sant Andreu di Barcellona. La preoccupazione della Generalità sulla questione degli alloggi porta alla creazione, nel 13 di giugno di 1932, del Comissariat de la Casa Obrera. Questo diventerà l'organo centrale per le opere della Generalità, dove parteciperanno tre dei principali rappresentanti del GATCPAC: Josep Torres Clavé, Joan Baptista Subirana e Josep Lluís Sert. Il nuovo organismo comissionerà, proprio a questi tre architetti, quello che diventerà il progetto più emblematico dell'edilizia abitativa nella Catalogna repubblicana: la Casa Bloc, il primo pezzo di quello che sarebbe stato il Piano di Urbanizzazione di Barcellona, noto come Piano Macià. L'edificio è stato costruito sulla stessa via Torres i Bages del quartiere di Sant Andreu, che mesi prima aveva promosso la prima costruzione di abitazioni per lavoratori della Generalità, occupando un intero isolato dell'estensione che aveva progettato il grande urbanista della Barcellona moderna, Ildefons Cerdà nel XIX secolo. La sua pianta, a forma di S, permette la distribuzione di 107 abitazioni su due piani accessibili da corridoi esterni. Il disegno della Casa Bloc riprende i criteri di Le Corbusier riguardo alla ventilazione e alla luce, l'ottimizzazione degli spazi interni e la liberazione dei piani terreni per dedicarli ad usi comunitari, come spazi culturali e spazi verdi. Ci si aspettava che questi spazi diventassero un asilo, una biblioteca, uno sport club e così via. La prima pietra del progetto è stata posta il 13 di marzo di 1933 e si è svolto il sorteggio delle abitazioni, che mai hanno finito di godere i suoi destinatari. La guerra civile sorprese il progetto nel mezzo della sua costruzione, progetto che poi fu ultimato senza seguire i criteri che

${ }^{8}$ AC. Documentos de Actividad Contemporánea, n. 25/1937, p. 34. 
l'avevano ispirato. Esso rimase incompleto, con l'aggiunta di spazi non previsti e l'occupazione delle zone verdi destinati ad usi molto diversi da quelli progettati.

A partire da quel momento saranno molti gli architetti, associati o sostenitori GATCPAC, che progetterano nelle loro città disegni di abitazioni seguendo le premesse stabilite dai teorici della nuova architettura. Nella città di Girona, l'architetto comunale, Ricard Giralt Casadesús, nel 1936, sviluppa l'urbanizzazione della montagna di Montjuïc con più di quaranta case per lavoratori, delle quali solo quattro andranno costruite insieme ad un blocco di abitazioni che non sarà mai costruito. Un altro architetto, associato al GATCPAC, Josep Claret Rubira, commissionato dal Comune, nel 1937 costruisce due progetti di abitazioni economiche. Una di queste costruzioni, le Case Escatllar, sono state considerate dalla storiografia un interessante contributo al dibattito dell'alloggio minimo e spesso appaiono negli studi sull'architettura del movimento moderno. Infine, un altro architetto, Joan Roca Pinet, anch'egli sotto commissione del governo della città, progetta un blocco de 16 abitazioni da affittare, che l'evoluzione del conflitto bellico impedisce nella loro realizzazione.

Un altro aspetto chiave del nuovo modello sociale promosso dalla Repubblica sarà quello di migliorare il sistema di istruzione. La carenza di edifici scolastici è stato un tema centrale, in maggiore o minore misura, di governatori e architetti di tutti i paesi europei. Negli anni trenta si riteneva improrogabile la necessità di migliorare i livelli di alfabetizzazione della popolazione e d'applicare i principi igienisti maturati dal XIX secolo. Il presidente Macià in un discorso nel 1931, spiegava con queste parole il programma di governo in materia scolastica:

Creeremmo qui una scuola in cui tutti i figli della Catalogna siano uguali di fronte al futuro della Cultura. Accanto a questa scuola sorgerà tutta una politica sociale [...] fatta di abitazioni in cui il figlio dell'operaio possa trovare godimenti, stimoli, spinte e comodità simili a quelli che il figlio del potente trova nella sua residenza. 9

Gli architetti rinnovatori, in risposta a questa dichiarazione programmatiche del governo, elaborano i principi di base sottostanti un edificio scolastico e si dedicano al disegno di progettazione per trasformare in realtà la volontà dei governanti. La nuova scuola deve essere un edificio economico e standardizzato, ricavato da una struttura modulare, con un buon orientamento e ventilazione e con una totale assenza di ornamenti e monumentalità.

\footnotetext{
9 ROVIRA, Josep M, SERRA, Carles, « Arquitectura Pública » in PIZZA, Antonio, ROVIRA, Josep M., G.A.T.C.P.A.C., op. cit., pp. 180-217.
} 
Uno dei primi progetti che è diventato realtà è stato quello della Escola de Palau Solità i Plegamans di Josep Lluís Sert, inaugurata nel 1933 e che grazie allo sforzo di standardizzazione è stata costruita con un $25 \%$ in meno rispetto al costo preventivato. A questo seguiranno molti progetti scritti dai membri del GATCPAC e dai suoi seguaci, in tutte le regioni della Catalogna. In un inventario che abbiamo elaborato recentemente sui gruppi scolastici costruiti nella Provincia di Girona dalla seconda metà del XIX secolo e fino al ripristino della Democrazia nel 1975, abbiamo constatato che un terzo dei 315 progetti documentati sono stati approvati durante gli otto anni della Repubblica. ${ }^{10}$ Cioè, la stessa percentuale di quelli costruiti in quasi quattro decenni della dittatura di Franco. Solo che più di un terzo dei progetti elaborati dalla Repubblica è rimasto nel cassetto, come conseguenza in quasi tutti i casi dello scoppio della Guerra Civile. Alcuni dati dimostrano che la storia dell'architettura scolastica è il riflesso del valore che i differenti governi hanno dato alla educazione. Per la Repubblica, l'architettura scolastica diventa una delle misure di governo più popolari e visibili nel territorio, ed è stata ampiamente descritta nella stampa locale come esempio dei nuovi tempi che il paese stava vivendo.

Seguendo la stessa politica di promozione del benessere dei cittadini e di collegamento con le tendenze più innovative a livello internazionale, il governo della Repubblica si propone di dotare il paese della necessaria infrastruttura sanitaria. Nel 1932 la Generalitat di Catalogna crea la Comissió Parlamentària de Sanitat i Assistència Social, con l'obiettivo di formulare il corpo legislativo che permettesse di progettare il sistema ospedaliero di Catalogna. In parallelo e con lo stesso obiettivo si da incarico al Sindicat de Metges de Catalunya, la redazione di diversi programmi di riforma e promozione del sistema sanitario. Uno dei risultati di questi lavori è la Llei de Divisió Sanitària de Catalunya e il tentativo di creare una rete di ospedali gerarchizzati in tre categorie secondo la vicinanza con l'utente.

Formulata l'organizzazione territoriale, occorreva declinare in concreto il campo dell'architettura ospedaliera. La maggior parte dei centri in attivo avevano un'origine antica ed erano giunte al secolo $\mathrm{XX}$ in precarie condizioni di conservazione, con utensili decrepiti e assegnazioni insufficienti di personale. Agli architetti rinnovatori furono prospettate nuovamente buone prospettive di collaborazione con il governo nella modernizzazione di alcune strutture qualificate di importanza strategica per il lavoro sociale che comportavano. All'inizio di settembre di 1934, Joan Baptista

${ }^{10}$ DOMÈNECH, Gemma, « Ús i abús del patrimoni arquitectònic escolar » in Revista de Girona, Girona, n. 232/2005, pp. 85-91. 
Subirana, architetto associato al GATCPAC, riceverà la commissione di riforma e ampliamento degli ospedali di Palafrugell, Badalona, Igualada, Manresa, Viella i Vic e successivamente la realizzazione di un progetto di un nuovo impianto standard per un ospedale di 100 letti che, in pratica, non sarà mai realizzato.

I nuovi edifici applicavano i principi terapeutici più avanzati come la struttura totalmente in metallo con gallerie vetrate situate a mezzogiorno, sale di trattamento dell' elioterapia, attrezzature moderne, ecc. Uno dei progetti simbolo del governo sarà il Dispensari Antituberculós nel quartiere del Raval di Barcellona. Commissionato nel 1933 agli architetti Josep Lluís Sert, Joan Baptista Subirana e Josep Torres Clavé, è diventato, insieme alla casa Bloc, una delle opere più emblematiche del periodo.

Il bilancio del periodo repubblicano nel campo dell'architettura sanitaria parla di molte opere di riforma e ampliamento in centri già esistenti in tutta la Catalogna. L'unica opera pubblica di nuovo impianto è il Dispensari Antituberculós de Barcelona. Il resto, le opere emerse dalla mappa sanitaria e dal piano direttore che è stato elaborato, sono state frenate dalla guerra civile. Gli architetti rinnovatori, associati o non associati al GATCPAC, avevano lavorato per dotare il paese di un sistema sanitario moderno ed efficiente, che garantisse un'ottima assistenza a tutti gli utenti. La guerra, tuttavia, ancora una volta, lascerà in sospeso i progetti.

\section{La 'fine del sogno'}

Durante il breve periodo repubblicano (1931-1939) questo gruppo di architetti lavora intensamente per trasformare in realtà il paese sognato da politici e architetti. Ma, all'avanzare del conflitto, si va configurando un panorama sconfortante per il progetto repubblicano. I problemi nella fornitura di materiali, derivanti della situazione bellica, lascerà nel cassetto qualche decina di progetti di rinnovatori: nella regione di Girona se ne contano $17^{11}$, e altri, già avviati, rimarranno bloccati e non saranno mai più ripresi. Un buon esempio è il Mercato Comunale di Olot, disegnato nel 1937, nel bel mezzo della guerra, da Bartomeu Agustí, architetto associato al GATCPAC. Le opere sono state sospese quando era stata costruita soltanto la struttura, ma, finita la guerra, la fazione vincitrice, nella volontà di cancellare ogni traccia dell'azione repubblicana, demolì l'opera incompiuta e ne costruì una nuova con un altro progetto.

Per gli architetti, la prospettiva non è delle migliori. Alcuni muoiono nel campo di battaglia, come Josep Torres Clavé, anima del movimento, che sulla scia degli

${ }^{11}$ Vedi: DOMÈNECH, Gemma, GIL, Rosa Maria, Un nou model d'arquitectura al servei d'una idea de país, Barcelona, Fundació Josep Irla - Duxelm, 2010. 
avvenimenti decide di lasciare il lavoro che svolgeva nella retroguardia ed andare al fronte per lavorare nelle fortificazioni, dove muore il 12 di gennaio di 1939. Gli altri vedranno come l'identificazione tra la Repubblica e il movimento innovativo dell'architettura, si tradurrà, finita la guerra, in rifiuto verso questo modello architettonico i cui membri andranno incontro alla repressione e all'esilio.

Come precedentemente affermato, a partire di 1939 il fascismo vincitore svolse una intensa attività repressiva e punitiva, con l'intenzione di cancellare il passato più vicino e di trovare nuovi seguaci per la nuova ideologia. Oltre alle migliaia di morti e di prigionieri dell'autoanominata giustizia di Franco, occorre segnalare una massiccia epurazione tra i professionisti che avevano lavorato per la Repubblica. Nel caso degli architetti, questa epurazione politico-sociale è effettuata dagli stessi collegi professionali e gli architetti coinvolti nel movimento rinnovatore saranno le principali vittime.

Alla fine di luglio di 1939, si costituirono nei sette collegi di architetti di Spagna le Comisiones de Depuración, che dovevano analizzare il comportamento dei loro associati secondo i parametri del nuovo regime. La normativa stabiliva che tutti i professionisti avrebbe dovuto rispondere ad una dichiarazione giurata sulla sua propria attività durante gli anni della Repubblica. Questa doveva essere verificata dalla Comisión, a partire dalla delazione da parte dei colleghi stessi e, successivamente, la Comisión proponeva la sanzione, secondo le norme previste, che andavano dal rimprovero privato all'inabilitazione all'esercizio della professione. ${ }^{12}$ Infine, sarebbe stato compito del Ministerio de la Gobernación, attraverso la Dirección General de Arquitectura, approvare o modificare le punizioni definitive. ${ }^{13}$

Tutto il procedimento si basava sull' «orden de 23 de febrero de 1940 dictando normas para la depuración de la conducta política y social de los Arquitectos », dove erano numerate con precisione le azioni imputabili di sanzioni e la natura di queste. ${ }^{\mathbf{1 4}}$ La prima recita: « todos los hechos de carácter profesional incursos en sanción de los Tribunales Militares o de Responsabilidades Políticas», ossia, qualsiasi condanna in un altro tribunale ne implicava automaticamente un'altra. La seconda: «la acceptación voluntaria, la obtención y desempeño de cargos profesionales durante la dominación marxista, cuyo carácter lucrativo o representativo pueda determinar afinidades ideológicas o políticas con el Frente Popular» o, detto altrimenti, aver lavorato per la

\footnotetext{
12 «Normas para la depuración de los arquitectes» dettate il 21 di luglio di 1939 per il Consejo Superior de los Colegios de Arquitectos de España.

${ }_{13}$ «Orden de 23 de febrero de 1940 dictando normas para la depuración de la conducta política y social de los Arquitectos», Boletín Oficial del Estado, n. 59/1940, pp. 1455-1457.

14 Ibidem.
} 
Repubblica. Sarebbero state inoltre motivo di punizione: «la contribución de cualquier orden a persecuciones o molestias contra otros colegiados, particulares o entidades profesionales» e «la utilización de influencias políticas, propias o ajenas, en propio beneficio, con daño moral o material para otros Colegiados o compañeros de profesión». Ugualmente veniva considerato delittuoso «la publicación de escritos contrarios al Movimiento Nacional o favorable a las doctrinas del Frente Popular, a sus partidarios o a sus actos; la firma de documentos beneficiosos a la revolución marxista, hechos ambos espontáneos y voluntarios» e «cualquier servicio positivo a la acción marxista judaica y anarquizante, en cualquiera de los sectores de la sociedad española, antes o después del Movimiento Nacional». Ed infine, «las acciones u omisiones que, sin estar expresamente comprendidas en los apartados anteriores, implicaran una evidente significación antipatriótica y contraria al Movimiento Nacional», ossia ciò che è soggettivamente considerato antipatriottico. Anche se non si fa esplicita mansione, sarebbe stato considerato delitto l'essere stato associato al GATCPAC. Ma, come vedremo, l'arbitrarietà della giustizia franchista lo prenderà in considerazione soltanto in determinati casi.

Analizzando i documenti conservati presso l'Archivio del Collegio di Architetti, sappiamo che nel mese di luglio di 1940 i membri della commissione epuratrice del collegio catalano inviano a Madrid due liste: quella dei «Señores Colegiados que no tienen ninguna nota desfavorable» con il nome di 163 architetti (che rappresentano il 70\%) e «Fichas de los restantes señores colegiados», dove ci sono 67 architetti (il $30 \%) .15$

Nel caso dei sospettati di cattivo comportamento, potevano comparire uno o più reati di imputazione. Di questi, la più comune è l' aver partecipato alla guerra nelle fila della fazione repubblicana (39 casi); seguita da quella di essere fuggito all'estero (19); l'avere preso parte alla direzione o all'organizzazione del Sindacato di Architetti durante la guerra (17); l'essere stato membro del GATCPAC (11), gruppo definito dagli oppressori come associazione di stampo comunista; l'avere avuto una visione di sinistra evidente senza specificare in nessun caso in che cosa consistesse (7); aver lavorato per il governo della Repubblica (7); ancora, l' essere stato condannato da altri tribunali o essere stato incarcerato o in un campo di concentramento (4); non avere presentato la dichiarazione giurata (2); l'avere avuto precedenti ascrivibili tra le ideologie di sinistra (2) o comunista (1), ecc.

Focalizzando l'attenzione sulla repressione subita dagli architetti affiliati al GATCPAC, bisogna segnalare che dei 81 membri iscritti ne saranno giudicati 45. I 36

\footnotetext{
15 Arxiu Històric del Col-legi d'Arquitectes de Catalunya. Fons Depuració Professional C-614/1.
} 
rimanenti non passaranno per il processo per essere studenti e per non avere esercitato sotto il piano professionale, perché non iscritti al collegio catalano, o per essere morti prima del 1939. I 45 interessati al processo di epurazione, vediamo come 15 figurino nella lista dei "Señores Colegiados que no tienen ninguna nota desfavorable », mentre i restanti 30 rientrano nella lista di quelli con imputazioni. Come possiamo vedere, nel caso di architetti associati con il movimento di rinnovamento, la percentuale di imputati è molto più elevata (il 66\%). In questi casi, l'accusa più frequente è rappresentata dall'essere stato ufficiale dell'esercito repubblicano (15), ma la seconda sarà l'essere stato associato al GATCPAC, reato che appare in 11 dei 18 casi. Seguono poi le accuse di fuga all'estero (9),l' avere lavorato per la Repubblica (6), l'avere sviluppato posizioni dirigenziali nel Sindacato di Architetti (5), l'avere avuto un atteggiamento di sinistra o comunista evidente (3), come anche l'avere antecedenti riconducibili alle aree della sinistra o in quella comunista (2.)

Tra il 5 agosto del 1940 e il 26 di marzo 1942 giungono a conclusione i 252 casi, che infine confluiscono nel collegio catalano (inizialmente erano 230). Alla fine del processo, la Junta de Depuración elabora due liste con i nominativi degli architetti catalani: la «Relación de Sanciones», che colpisce ventisei architetti, e la «Relación de arquitectos depurados sin sanción», che interessa 207 architetti. Ai sanzionati occorre aggiungere i diciannove che il 26 di marzo 1942 sono stati sospesi dalla pratica professionale privata e pubblica per «encontrarse huidos en el extranjero». ${ }^{16}$

Analizzando singolarmente i casi (imputazioni e condanne), possiamo concludere che il collegio si è limitato a fare un trasposizione della repressione politica nell'ambito professionale. Bartomeu Agustí, associato al GATCPAC, che era stato condannato dal Tribunal de Responsabilidades Políticas a dodici anni di prigione, ricevette una delle sanzioni più alte che poteva imporre il collegio: la sospensione totale dell'esercizio pubblico e privato della professione. La stessa sanzione che ricevette Emili Blanch, condannato dal Tribunal de Responsabilidades Políticas alla «incautación total de bienes, inhabilitación absoluta en su grado máximo y relegación a las posesiones africanas por el plazo de quince años». Nel frattempo, gli architetti che avevano esplicitato pubblicamente la loro adesione al nuovo regime furono rinviati a processo in pochi giorni e assolti senza penalità o, al massimo, con un semplice rimprovero, come nel caso di Josep Claret, che dopo essere stato assolto nel processo militare respinge le accuse del tribunale professionale riferendosi alla sua situazione familiare:

16 Ibidem. 
Mal se compagina la acusación [di essere di sinistra, anti-religioso o catalanista] con el hecho de que contrajo matrimonio en el año 1934 con la hija de familia tan reputada y conocida por su acendrado españolismo y catolicismo, como es la de Don Prudencio Rodríguez Chamorro, que fué Gobernador Civil de la Provincia de Gerona, durante la Dictadura, en cinco años, y con el Glorioso Movimiento Alcalde de Zamora. ${ }^{17}$

Troppo spesso i processi rendono evidente un'applicazione delle misure disciplinari condizionata dai rapporti, affinità e divergenze personali, una caratteristica, questa, insita nel modello di giustizia caricata d'arbitrarietà del nuovo regime. Un buon esempio è la questione dell'affiliazione al GATCPAC, che appare soltanto in 11 dei 30 processi aperti contro i membri del gruppo e, non sempre viene riferita come imputazione per i membri con più responsabilità del gruppo. Non si può parlare, quindi, di una epurazione professionale ma piuttosto di un asettico adattamento del collegio al nuovo regime.

I rappresentanti della nuova architettura morti, vittime di rappresagli o esiliati, rimarranno definitivamente dispersi. I loro progetti interrotti, come il Pla Macià, deprezzati, come la Casa Bloc o semplicemente archiviati, come molte scuole e strutture in tutti i villaggi di Catalogna. Ma la maggioranza degli architetti che sono sopravvissuti non hanno mai dimenticato l'esperienza. Il razionalismo come stile e la funzione sociale dell'architettura come atteggiamento, compariranno timidamente nei progetti che essi redigeranno quando, negli anni cinquanta, sarebbero state eliminate le sanzioni d'inabilitazione. Ma la società che circonda coloro che erano rimasti o coloro che tornarono dall'esilio, come nel caso di Emili Blanch, impedisce loro di tornare a godere del prestigio sociale e professionale che avevano prima della guerra. Settanta anni dopo, alcune delle idee ancora presenti nell'architettura e nell'urbanistica trovano le loro origini nell'esperienza di quei giovani architetti, toccati dall'utopia e ridotti al silenzio dalla brutalità di una guerra civile.

17 Ibidem. 


\section{* L'autore}

Gemma Domènech Casadevall è dottore di Ricerca in Storia dell'Arte presso l'Universitat Autònoma de Barcelona (2000). Ha quindi svolto un post-dottorato nel Institut Català de Recerca en Patrimoni Cultural (ICRP - Istituto Catalano di Ricerca in Patrimonio Culturale). Ha pubblicato diversi saggi sul patrimonio dell'epoca moderna e sull'architettura e gli architetti catalani della prima metà del secolo XX. Ha pubblicato, fra altri testi: «Fragile heritages: an architecture between disappearance and reutilization», in AMOÊDA, Rogério, LIRA, Sérgio, PINHERIO, Cristina, Heritage 2010. Heritage and sustainable development, Barcelos [Portugal], Green Lines Institute for Sustainable Development, 2010, p.837-844; «Republicanisme i renovació arquitectònica», in PUJOL, Enric, El somni republicà. El republicanisme a les comarques gironines [190o-1936], Girona, Ed. Viena-Diputació de Girona, 2009; con Carles Ribera: "Camins de sortida. L'exili gironí del 1939 » In Girona 1939-1953. Exilis, repressions i complicitats, Girona, Ajuntament de Girona, 2009; e con Rosa María Gil: nou model d'arquitectura al servei d'una idea de país. Barcelona, Fundació Josep Irla - Duxelm, 2010; e Josep Claret Rubira: arquitecte entre la República i la dictadura, Girona, Ajuntament de Girona - COAC, 2009.

URL: < http://studistorici.com/progett/autori/\#DomènechCasadevall >

\section{Per citare questo articolo:}

DOMĖNECH CASADEVALL, Gemma, "Guerra civile e architettura spagnola moderna: la fine di un sogno», Diacronie. Studi di Storia Contemporanea: Spagna Anno Zero: la guerra come soluzione, 29/07/2011,

URL:< http://www.studistorici.com/2011/07/29/domenech_numero_7/>

\section{Diacronie Studi di Storia Contemporanea $\beta$ www.diacronie.it}

Risorsa digitale indipendente a carattere storiografico. Uscita trimestrale.

redazione.diacronie@hotmail.it

Comitato di redazione: Marco Abram - Giampaolo Amodei - Jacopo Bassi - Luca Bufarale - Alessandro Cattunar - Alice De Rensis Barbara Galimberti - Deborah Paci - Fausto Pietrancosta - Martina Sanna - Matteo Tomasoni - Luca Zuccolo

Diritti: gli articoli di Diacronie. Studi di Storia Contemporanea sono pubblicati sotto licenza Creative Commons 2.5 Possono essere riprodotti a patto di non modificarne i contenuti e di non usarli per fini commerciali. La citazione di estratti è comunque sempre autorizzata, nei limiti previsti dalla legge. 